\title{
Adaptive leaderless consensus of agents in jointly connected networks
}

\author{
Hui $\mathrm{Yu}^{\mathrm{a}, *}$, Xiaohua Xia ${ }^{\mathrm{b}}$ \\ ${ }^{a}$ College of Science, China Three Gorges University, Yichang 443002, China \\ ${ }^{b}$ Centre of New Energy Systems, Department of Electrical, Electronics and Computer \\ Engineering, University of Pretoria, Pretoria 0002, South Africa
}

\begin{abstract}
In this paper, the leaderless consensus problem of multi-agent systems with jointly connected topologies and nonlinear dynamics is considered, in which the nonlinear dynamics are assumed to be non-identical and unknown. The unknown nonlinear dynamics existing in the systems are assumed to be linearly parameterized, and an adaptive design method for leaderless multiagent systems is presented. By just using the relative position information between each agent and its neighbours, a distributed adaptive consensus control algorithm for the considered systems is proposed, in which the network graphs are jointly connected. Both the global uniform asymptotical stability and the global uniform asymptotical parameter convergence analysis of the adaptive control algorithm are carried out by using adaptive control theory, Lyapunov theory and algebraic graph theory. Finally, an example is given to illustrate the validity of our theoretical results.
\end{abstract}

Keywords: Adaptive consensus, Decentralized control, Parameter convergence, Jointly connected topology, Multi-agent system

\section{Introduction}

Distributed cooperative control of multi-agent systems has been taken much attentions by many researchers in automatical control and multi-robot coordination. Its broad applications include in various fields, such as, multi-

\footnotetext{
${ }^{*}$ Corresponding author.

Email addresses: yuhui@ctgu.edu.cn (Hui Yu), xxia@up.ac.za (Xiaohua Xia)
} 
robot rendezvous, flocking and swarming control, multi-robot formation control, etc. In distributed coordinated control of muliti-agent systems, a significant problem is to design distributed coordinated controllers such that consensus can be achieved on a decision value.

In the past decades, the consensus problem of both leader-following and leaderless multi-agent systems have been extensively studied on different topics under various assumptions, for example, distributed robust consensus [1], consensus in time-delay networks $[2,3]$, consensus with finite-time convergence [4], average consensus [5, 6], consensus in jointly connected networks [7], group consensus [8], containment control [9], consensus with communication constraints [10], etc. In the studies of consensus problem of multi-agent systems, variable network topologies have been extensively investigated in the literature. However, information transfer may interrupt in some practical applications due to the instability of communication channels. Therefore, joint connectedness $[7,11,12]$ is an important assumption for the network topologies of multi-agent systems. It does not requires that the switching graphs are connected at any moment, which means that the switching graphs are permitted to be disconnected at any time instant.

In practice, uncertainty and unmodeled dynamics may exist in the systems. Therefore, one of the interesting topics on consensus problem is investigating the case of multi-agent systems with unknown nonlinearity. The adaptive design method is a good choice. In [13, 14], a coordination problem steering a group of agents to a formation with a prescribed reference velocity is considered. Adaptive algorithms are proposed for reference velocity recovery [13] and reference velocity tracking [14]. In [15], robust adaptive design techniques is applied in multi-agent systems such that the considered systems can reach consensus. As the controller for each agent only use its neighbor agents' information, the proposed algorithm is distributed. In [16], the authors propose an adaptive finite-time leader-following consensus algorithm for multi-agent networks, in which the model dynamics of both leader and follower agents are non-identical, unknown and nonlinear. A type of homogenous Lyapunov function is introduced in the finite-time control algorithm design and stability analysis based on finite-time stability theory. In [17], the unknown nonlinear dynamics of multi-agent systems are approximated by neural networks. An adaptive controller is proposed for directed multi-agent networks such that the consensus error vectors and weight estimate errors are uniformly ultimately bounded. In [18], for a class of interconnected nonlinear pure-feedback systems, the unknown nonlinear 
dynamics of the interconnected system are approximated by fuzzy logic systems. An adaptive output feedback control approach is proposed such that all variables are semi-globally uniformly ultimately bounded and the system errors converge to a small neighborhood of the origin. In [19], under linear parameterizations assumptions, an adaptive control strategy is proposed for leader-following multi-agent networks that is undirected and jointly connected. In [20], the authors present distributed cooperative adaptive controllers to solve the uniformly exponential stability problem of a group of uncertain systems in a general framework. Some other studies on adaptive consensus of multi-agent systems focus on adaptive tuning of the network weights, or coupling strength [21, 22].

In this paper, the leaderless consensus problem of multi-agent systems is studied. The multi-agent networks considered in this paper are jointly connected. The system model is assumed to be nonidentical, nonlinear and unknown. The uncertain dynamics of all agents are assumed to be linearly parameterized by some basis functions and the unknown parameters are estimated by each agent. For networks with jointly connected topologies, a distributed adaptive consensus scheme is proposed through only relative position feedback between agents. By introducing the Persistent excitation $(\mathrm{PE})$ assumption for regressor matrix, and using algebraic graph theory and Lyapunov techniques, we prove that the consensus can be achieved globally uniformly asymptotically, in the meanwhile, the global uniform asymptotical parameter convergence to zero is also guaranteed.

The main contributions of this work are mainly in three aspects. First, a purely distributed adaptive consensus algorithm is proposed for leaderless multi-agent networks which are assumed to be jointly connected. Consensus analysis is given by using Lyapunov theory, algebraic graph theory, and Barbalat's lemma. In [19], a similar model is considered for leader-following multi-agent systems. The control algorithm proposed in [19] depends on the local consensus errors from itself and its neighbors. From the definition of the local consensus error, each agent requires not only the information of its neighbors but also the information of its neighbors' neighbors. Therefore, it is not purely distributed. In this paper, a purely distributed control algorithm only depending on relative position measurements between its neighbors, is proposed. The leaderless consensus stability analysis is more challenging than that in leader-following systems, because the zero eigenvalue of the system matrix in leaderless systems is intrinsic even if the graph is connected. Second, by introducing the PE condition, a sufficiency condition is derived for 
the considered systems to achieve the parameter convergence. Since the intrinsic zero eigenvalue exists in leaderless multi-agent systems, the parameter convergence analysis is a challenging work. By using some transformations, a common matrix $\mathrm{C}$ is obtained to derive the sufficiency condition for parameter convergence. The $\mathrm{PE}$ condition is also introduced in $[13,14,20]$ for the parameter convergence. In $[13,14]$, because the topology of interconnected graph is fixed, the parameter convergence is straightforward form the classical adaptive control theory. In [20], a cooperative PE condition and a integration-based topology condition are introduced for cooperative adaptive systems to reach uniformly exponential stability in a general framework. Some applications are given for identification and control of multi-agent systems. Third, the topologies of the interconnected graphs are switching, especially, jointly connected. This condition is more general. Except for [19, 20], the works $[13-18,21,22]$ on adaptive system mentioned above are all for networks with fixed topology.

This paper is built up as follows. We state formally the problem which is formulated with some notations in Section 2. We present our main results in section 3. We give an illustrative example and show the simulation results to validate our theoretical results in section 4 . Concluding remarks are given in section 5 .

\section{Preliminaries and Problem Statement}

\subsection{Preliminaries}

The multi-agent interconnection networks can be expressed by graphs. A graph $\mathscr{G}(\mathscr{V}, \mathscr{E})$ consists of a node set $\mathscr{V}=\{1,2, \cdots, N\}$ and an edge set $\mathscr{E} \subset \mathscr{V} \times \mathscr{V}$, in which an edge $(i, j)$ in the edge set $\mathscr{E}$ is an unordered pair. A simple graph is undirected, and has no repeated edges and self-loops. In this paper, only simple graphs is considered. In an edge $(i, j)$, the node $j$ is termed as neighbor of the node $i$. Denote neighbors set of node $i$ by $\mathscr{N}_{i}=\{j \in \mathscr{V} \mid(i, j) \in \mathscr{E}, j \neq i\}$. A path in a graph is a sequence of edges $(1,2),(2,3), \cdots$ in the graph. A graph $\mathscr{G}$ is termed to be connected if there exists a path between any two nodes of the graph. For a collection of graphs, its union is defined as a new graphs where its node set and edge set are the union of node set and edge set of all of the graphs in the collection. If the union of a collection of graphs is connected, we say that the collection of graphs is jointly connected. 
The information exchanges between agents can be modelled by a graph $\mathscr{G}$, in which each agent is according to a node in $\mathscr{V}$. An edge $(i, j)$ means that the agent $i$ can receive, obtain or sense information from agent $j$. Denote the weighted adjacency matrix of a graph $\mathscr{G}$ by $A=\left[a_{i j}\right] \in \mathcal{R}^{N \times N}$, where $a_{i j}>0$ if $(i, j) \in \mathscr{E}$ and $a_{i j}=0$ if $(i, j) \notin \mathscr{E}$. The degree of node $i$ in a graph is defined as $\sum_{j \in \mathscr{N}_{i}} a_{i j}$. The degree matrix $D \in \mathcal{R}^{N \times N}$ of a graph $\mathscr{G}$ is a diagonal matrix whose $i$ th entry being the degree of node $i$. Let $L=D-A$, the matrix $L$ is called the Laplacian of the graph. The following lemma of $L$ in algebraic graph theory [23] is well known.

Lemma 1. The Laplacian $L$ of graph $\mathscr{G}$ has at least one zero eigenvalue with $\mathbf{1}_{N}=(1,1, \cdots, 1)^{T} \in R^{N}$ as its eigenvector, and all the non-zero eigenvalues of $L$ are positive. The Laplacian $L$ has a simple zero eigenvalue if and only if graph $\mathscr{G}$ is connected.

In this paper, we consider the case that the interconnected graphs of the systems are switching over time. The set of all possible switching graphs is denoted by $\left\{\mathscr{G}_{p} \mid p \in \mathscr{P}\right\}$, where $\mathscr{P}$ is an index set. For describing the time dependence of graphs, a piecewise constant switching signal $\sigma(t):[0, \infty) \rightarrow$ $\mathscr{P}$ is defined. We use $\mathscr{G}_{\sigma(t)}$ to denote the underlying graphs at time $t$ on $N$ nodes. Since the Laplacian $L$ of the graph, the neighbors set $\mathscr{N}_{i}$ of agent $i$, and the $(i, j)$ th entry $a_{i j}$ of $A$ are all vary with time, their time varying versions are denoted by $L_{\sigma(t)}, a_{i j}(t)$ and $\mathscr{N}_{i}(t)$, respectively.

\subsection{Problem Statement}

Consider a group of $N$ nonidentical nonlinear agents, the dynamics of the $i$ th agents are described by

$$
\dot{x}_{i}(t)=f_{i}\left(x_{i}(t), t\right)+u_{i}(t), \quad i=1,2, \cdots, N,
$$

where $x_{i}(t) \in \mathcal{R}$ is the position state of the $i$ th agent, $u_{i}(t) \in \mathcal{R}$ is the control input, and $f_{i}\left(x_{i}(t), t\right)$ is the unknown nonlinear dynamics of agent $i$, which is assumed to be continuous in $t$ and Lipschitz in $x_{i}(t)$ to guarantee the existence of unique solution of equation (1).

We denote the stack column vector of $x_{i}(t)$ with $i$ in some index set $S$ by $\operatorname{col}\left(x_{i}(t)\right)_{i \in S}$ or simply $\operatorname{col}\left(x_{i}(t)\right)$, the stack column vector of vector $x$ and $y$ by $\operatorname{col}(x, y)$, etc. Letting $x(t)=\operatorname{col}\left(x_{i}(t)\right), u(t)=\operatorname{col}\left(u_{i}(t)\right), f(x, t)=$ $\operatorname{col}\left(f_{i}\left(x_{i}, t\right)\right)$, the dynamics of $N$ agents can be rewritten as

$$
\dot{x}(t)=f(x, t)+u(t) .
$$


Remark 1. We assume that $x_{i} \in \mathcal{R}, i=1,2, \cdots, N$, to avoid complicated expressions. It is trivial to extend this case to that of $x_{i} \in \mathcal{R}^{n}, i=1,2, \cdots, N$, by using Kronecker product $\otimes$.

In this work, the consensus problem of above systems is considered, that is, designing distributed controllers $u_{i}(t), i=1,2, \cdots, N$, such that the consensus can be reached.

For the considered systems (1), the consensus is said to be achieved if, for some distributed controller $u_{i}(t), i=1,2, \cdots, N$,

$$
\lim _{t \rightarrow \infty}\left|x_{i}(t)-x_{j}(t)\right|=0, \quad i \neq j, \quad i, j=1,2, \cdots, N,
$$

for $\forall x_{i}(0), i=0,1, \cdots, N$.

\section{Main results}

In this section, the linear parameterizations models of the leaderless systems (1) are given firstly. Then, we present our distributed controllers and parameter adaptive laws. Finally, the stability and parameter convergence analysis are given.

The unknown nonlinear dynamics $f_{i}\left(x_{i}(t), t\right), i=1, \cdots, N$, of group of agents are supposed to be linearly parameterized as

$$
f_{i}\left(x_{i}(t), t\right)=\phi_{i}^{T}\left(x_{i}(t), t\right) \vartheta_{i}, \quad i=1,2, \cdots, N,
$$

where $\phi_{i}\left(x_{i}(t), t\right) \in \mathcal{R}^{m}$ is the basis function column vector, $\vartheta_{i} \in \mathcal{R}^{m}$ is the parameter column vector which is constant, unknown and will be estimated.

Let $\hat{\vartheta}_{i}$ be the estimate of $\vartheta_{i}$, the estimate of $f_{i}\left(x_{i}(t), t\right)$ can be expressed as

$$
\hat{f}_{i}\left(x_{i}(t), t\right)=\phi_{i}^{T}\left(x_{i}(t), t\right) \hat{\vartheta}_{i}, \quad i=1,2, \cdots, N .
$$

Remark 2. The linearly parameterized models of the unknown nonlinear $d y$ namics have been extensively investigated in classical adaptive control theory [24, 25]. Examples for multi-agents systems can be found in [13, 14, 16, 19, 26].

\subsection{Distributed adaptive consensus algorithm design}

For multi-agent systems (1), let $\left[t_{k}, t_{k+1}\right), k=0,1,2, \cdots$ be an infinite time interval sequence, where $t_{0}=0$ is the initial time instant, $T_{0} \leq t_{k+1}-$ $t_{k} \leq T$, and $T_{0}, T$ are some positive constants. 
Assumption 1. The switching times of the switching signal $\sigma(t)$ is finite in any bounded time intervals.

From Assumption 1, suppose that each time interval $\left[t_{k}, t_{k+1}\right), k=0,1,2, \cdots$, is divided into a finite time interval sequence

$$
\left[t_{k}^{0}, t_{k}^{1}\right), \ldots,\left[t_{k}^{l}, t_{k}^{l+1}\right), \cdots,\left[t_{k}^{l_{k}-1}, t_{k}^{l_{k}}\right),
$$

where $t_{k}=t_{k}^{0}, t_{k+1}=t_{k}^{l_{k}}$ for some integer $l_{k} \geq 0, t_{k}^{l+1}-t_{k}^{l} \geq \tau, 0 \leq l \leq l_{k}-1$, $\tau>0$ is a positive constant, the so-called dwell time. $t_{k}^{0}, t_{k}^{1}, \cdots, t_{k}^{l_{k}-1}$ are the time instants at which the time-varying graph switches, in other words, in each such time subinterval $\left[t_{k}^{l}, t_{k}^{l+1}\right)$ the switching graph $\mathscr{G}_{\sigma(t)}$ is time invariant. Note that the switching graph $\mathscr{G}_{\sigma(t)}$ is possibly disconnected in each such subinterval $\left[t_{k}^{l}, t_{k}^{l+1}\right)$. If the union of $\left\{\mathscr{G}_{\sigma(t)} \mid t \in\left[t_{k}, t_{k+1}\right)\right\}$ is connected, then we say it is jointly connected across the time interval $\left[t_{k}, t_{k+1}\right), k=0,1,2, \cdots$.

Motivated by [12], we have the following lemma:

Lemma 2. Let matrices $L_{i_{1}}, L_{i_{2}}, \cdots, L_{i_{s}}$ be associated with the graphs $\mathscr{G}_{i_{1}}, \mathscr{G}_{i_{2}}$, $\cdots, \mathscr{G}_{i_{s}}$, respectively. If these graphs are jointly connected, then $\sum_{p=i_{1}}^{i_{s}} L_{p}$ is an effective Laplacian matrix of some connected graph.

Proof. Obviously, matrix $\sum_{p=i_{1}}^{i_{s}} L_{p}$ is an effective Laplacian matrix of the connected graph $\cup_{p=i_{1}}^{i_{s}} \mathscr{G}_{p}$.

Assumption 2. In each time interval $\left[t_{k}, t_{k+1}\right)$, the switching graph $\mathscr{G}_{p}, p \in$ $\mathscr{P}$ is jointly connected.

Remark 3. In the study of switching topologies, the joint connectedness [7, 11, 12] subject to the Assumption 1 and 2 is more general. It does not require the switching graphs are connected at any moment. In other word, the switching graphs can be disconnected at any time instants.

Let $\Theta=\operatorname{col}\left(\vartheta_{i}\right), \hat{\Theta}=\operatorname{col}\left(\hat{\vartheta}_{i}\right)$. In the following, we present our control algorithm for adaptive consensus achieving:

$$
\begin{aligned}
u_{i}(t)= & -c \sum_{j \in \mathscr{N}_{i}(t)} a_{i j}\left[x_{i}(t)-x_{j}(t)\right]-\phi_{i}^{T}\left(x_{i}, t\right) \hat{\vartheta}_{i} \\
& i=1,2, \cdots, N,
\end{aligned}
$$

and

$$
\begin{aligned}
\dot{\hat{\vartheta}}_{i}(t)= & c_{1} \phi_{i}\left(x_{i}, t\right) \sum_{j \in \mathscr{N}_{i}(t)} a_{i j}\left[x_{i}(t)-x_{j}(t)\right], \\
& i=1,2, \cdots, N
\end{aligned}
$$


where $c, c_{1}>0$ are positive constant numbers.

The equations (7) and (8) can be rewritten in the following form:

$$
u(t)=-c L_{\sigma(t)} x(t)-\Phi^{T}(x, t) \hat{\Theta}
$$

and

$$
\dot{\hat{\Theta}}(t)=c_{1} \Phi(x, t) L_{\sigma(t)} x(t),
$$

where $\Phi(x, t)=\operatorname{diag}\left(\phi_{1}\left(x_{1}, t\right), \phi_{2}\left(x_{2}, t\right), \cdots, \phi_{N}\left(x_{N}, t\right)\right), L_{\sigma(t)}$ is the Laplacian of graph $\mathscr{G}_{\sigma(t)}$.

\subsection{Stability analysis}

Let $\bar{x}(t)=\frac{1}{N} \sum_{i=1}^{N} x_{i}(t)$ and define

$$
e_{i}(t)=x_{i}(t)-\bar{x}(t), \quad i=1,2, \cdots, N,
$$

as the consensus error of the $i$ th agent.

Let $e(t)=\operatorname{col}\left(e_{i}(t)\right)$,we have

$$
e(t)=\left(I_{N}-\frac{1}{N} 1_{N} 1_{N}^{T}\right) x(t)=C x(t)
$$

where $C=I_{N}-\frac{1}{N} 1_{N} 1_{N}^{T}=\left(\begin{array}{cccc}\frac{N-1}{N} & -\frac{1}{N} & \cdots & -\frac{1}{N} \\ -\frac{1}{N} & \frac{N-1}{N} & \cdots & -\frac{1}{N} \\ \vdots & \cdots & \cdots & \vdots \\ -\frac{1}{N} & -\frac{1}{N} & \cdots & \frac{N-1}{N}\end{array}\right)$ is positive semidefinite with eigenvalues 0 and $1, I_{N} \in \mathcal{R}^{N \times N}$ is the identity matrix.

Let $\bar{\vartheta}_{i}=\hat{\vartheta}_{i}-\vartheta_{i}, \bar{\Theta}=\hat{\Theta}-\Theta=\operatorname{col}\left(\bar{\vartheta}_{i}\right)$. Considering the time interval $\left[t_{k}^{l}, t_{k}^{l+1}\right), 0 \leq l \leq l_{k}-1, k=0,1, \cdots$, we have

$$
\begin{aligned}
\dot{e}(t) & =C \dot{x} \\
& =C[f(x, t)+u(t)] \\
& =-c C L_{\sigma(t)} e(t)-C \Phi^{T}(x, t) \bar{\Theta} .
\end{aligned}
$$

From (10)-(13) and the properties of $L$ in Lemma 1, we have

$$
L C=L-\frac{1}{N} L 1_{N} 1_{N}^{T}=L, C L=L-\frac{1}{N} 1_{N} 1_{N}^{T} L=L,
$$

then $L C=C L=L$. The error system of the system (1) can be obtained as follows:

$$
\left\{\begin{array}{l}
\dot{e}(t)=-c L_{\sigma(t)} e(t)-C \Phi^{T}(x, t) \bar{\Theta}, \\
\dot{\bar{\Theta}}(t)=c_{1} \Phi(x, t) L_{\sigma(t)} e(t) .
\end{array}\right.
$$


Let $\lambda_{\min }(P)$ be the the smallest non-zero eigenvalue of the positive semidefinite matrix $P$. The $N$ eigenvalues of $L_{p}$ are denoted by $\lambda_{p}^{1}, \lambda_{p}^{2}, \cdots, \lambda_{p}^{N}$ with $\lambda_{p}^{1}=0$ due to $L_{p}$ having at least a zero eigenvalue. Define $\gamma_{p}=\lambda_{\min }\left(L_{p}\right)$, $\mu_{p}=\lambda_{\max }\left(L_{p}\right), p \in \mathscr{P}$. Then

$$
\delta_{\min }=\min \left\{\gamma_{p} \mid p \in \mathscr{P}\right\}, \delta_{\max }=\max \left\{\mu_{p} \mid p \in \mathscr{P}\right\}
$$

are well defined and obviously positive.

Assumption 3. The PE (persistently exciting) condition [25]

$$
\int_{t}^{t+\delta_{0}} \Phi C \Phi^{T} d \tau \geq \alpha I>0, \quad \forall t \geq 0
$$

is satisfied, where $\delta_{0}$ and $\alpha$ are some positive reals.

Remark 4. In classical adaptive control theory [25], the PE condition is a standard assumption for the regressor matrix $\Phi$. It guarantees the information richness of the regressor $\Phi$ and plays a key role in the parameter convergence analysis, i.e.,

$$
\lim _{t \rightarrow \infty}\left\|\hat{\vartheta}_{i}-\vartheta_{i}\right\|=0
$$

for $\forall \hat{\vartheta}_{i}(0), i=1,2, \cdots, N$. However, the problem how to check the PE condition is still open. Because $C$ is symmetric and positive semi-definite, there exists some matrix $B$ such that $C=B B^{T}$. If $\Phi$ is periodic and then $\Phi B$ is also periodic. Additional conditions can be imposed which ensure the PE condition. For example, if $\Phi$ is periodic, then it is well known [24, 27] that the PE condition (17) is ensured.

In the following, we present our main result:

Theorem 1. Assume that the Assumption 1-3 are satisfied and suppose that $\phi_{i}$ and $\dot{\phi}_{i}, i=0,1, \cdots, N$, are uniformly bounded. Then, under the control scheme (7) and (8), the consensus of the multi-agent systems (1) can be reached globally uniformly asymptotically, and the global uniform asymptotical parameter convergence is also guaranteed in the sense of (3) and (18), respectively.

Proof. Considering the positive semi-definite function

$$
V(t)=\frac{1}{2 c} e(t)^{T} L_{p} e(t)+\frac{1}{2 c c_{1}} \bar{\Theta}^{T}(t) \bar{\Theta}(t) .
$$


for systems (15). Except for the switching instants, $V(t)$ is continuous and differentiable .

Consider a time interval $\left[t_{k}^{l}, t_{k}^{l+1}\right]$ with switching graph $\mathscr{G}_{p}, p \in \mathscr{P}$, included in the time interval $\left[t_{k}, t_{k+1}\right]$. Calculating the time derivative of $V(t)$ defined in (19) along the trajectory of the system (15), we get

$$
\begin{aligned}
\dot{V}(t) & =-e(t)^{T} L_{p}^{2} e(t) \\
& \leq-\gamma_{p} e(t)^{T} L_{p} e(t) \\
& \leq-\delta_{\min } e(t)^{T} L_{p} e(t) \\
& \leq 0 .
\end{aligned}
$$

Therefore, $V(t)$ is non-increasing and $V(t) \geq 0$. Then, $\lim _{t \rightarrow \infty} V(t)=$ $V(\infty)$ is finite.

In the following, we focus on proving $\lim _{t \rightarrow \infty}\left|x_{i}-x_{j}\right|=0$.

Consider the infinite sequence

$$
\left\{V\left(t_{k}\right), k=0,1, \cdots\right\} .
$$

Based on the Cauchy's convergence criteria, for $\forall \epsilon>0$, there exists an integer $K>0$, such that

$$
\left|V\left(t_{k+1}\right)-V\left(t_{k}\right)\right|<\epsilon
$$

for $\forall k>K$. Then, we have $\left|\int_{t_{k}}^{t_{k+1}} \dot{V}(t) d t\right|<\epsilon$ and therefore,

$$
\sum_{l=0}^{l_{k}-1} \int_{t_{k}^{l}}^{t_{k}^{l+1}} \dot{V}(t) d t>-\epsilon .
$$

From (20), we have

$$
\begin{aligned}
-\epsilon & <\sum_{l=0}^{l_{k}-1} \int_{t_{k}^{l}}^{t_{k}^{l+1}} \dot{V}(t) d t \\
& \leq-\sum_{l=0}^{l_{k}-1} \int_{t_{k}^{l}}^{t_{k}^{l+1}} \delta_{\min } e(t)^{T} L_{\sigma\left(t_{k}^{l}\right)} e(t) d t .
\end{aligned}
$$

Because $l_{k}$ is assumed to be finite within the time interval $\left[t_{k}, t_{k+1}\right)$. Then, we derive

$$
\begin{aligned}
-\epsilon & \leq-\delta_{\min } \int_{t_{k}^{l}}^{t_{k}^{l+1}} e(t)^{T} L_{\sigma\left(t_{k}^{l}\right)} e(t) d t \\
& \leq-\delta_{\min } \int_{t_{k}^{l}}^{t_{k}^{l}+\tau} e(t)^{T} L_{\sigma\left(t_{k}^{l}\right)} e(t) d t
\end{aligned}
$$

or equivalently

$$
\delta_{\min } \int_{t_{k}^{l}}^{t_{k}^{l}+\tau} e(t)^{T} L_{\sigma\left(t_{k}^{l}\right)} e(t) d t \leq \epsilon,
$$


for $\forall k>K$, where $l=0,1, \cdots, l_{k}-1$ and $k=0,1,2, \cdots$.

From (23) and $\epsilon>0$ being arbitrary, we have

$$
\lim _{t \rightarrow \infty} \int_{t}^{t+\tau} e^{T}\left(\sum_{l=0}^{l_{k}-1} L_{\sigma\left(t_{k}^{l}\right)}\right) e d s=0 .
$$

Now, we need to prove the function $e^{T}\left(\sum_{l=0}^{l_{k}-1} L_{\sigma\left(t_{k}^{l}\right)}\right) e$ is uniformly continuous. This is due to the uniform boundedness of $e(t), \bar{\Theta}(t)$ and $\dot{e}$, which can be derived form (15), (19), (20) and the assumption of uniform boundedness of $\phi_{i}$ and $\dot{\phi}_{i}$. From the Barbalat's lemma, we have

$$
\lim _{t \rightarrow \infty} e^{T}\left(\sum_{l=0}^{l_{k}-1} L_{\sigma\left(t_{k}^{l}\right)}\right) e=0 .
$$

Since the switching graphs are jointly connected across the time interval $\left[t_{k}, t_{k+1}\right)$, we have the matrices $\sum_{l=0}^{l_{k}-1} L_{\sigma\left(t_{k}^{l}\right)}, k=0,1,2, \cdots$, are effective Laplacian of some connected graph based on Lemma 2. Let $W_{k}=$ $\left[\frac{1}{\sqrt{N}} \mathbf{1}_{N}, S_{k}\right] \in \mathcal{R}^{N \times N}$ be an orthogonal matrix, and $S_{k} \in \mathcal{R}^{N \times(N-1)}$ such that

$$
W_{k}^{T}\left(\sum_{l=0}^{l_{k}-1} L_{\sigma\left(t_{k}^{l}\right)}\right) W_{k}=\operatorname{diag}\left\{\nu_{k}^{1}, \nu_{k}^{2}, \cdots, \nu_{k}^{N}\right\} \text {, }
$$

where $\nu_{k}^{1}=0,0<\nu_{k}^{2} \leq \cdots \leq \nu_{k}^{N}$, are the $N$ eigenvalues of $\sum_{l=0}^{l_{k}-1} L_{\sigma\left(t_{k}^{l}\right)}$, due to the symmetry of $\sum_{l=0}^{l_{k}-1} L_{\sigma\left(t_{k}^{l}\right)}$ and Lemma 1 .

Let $\zeta(t)=W_{k}^{T} e(t)$, we have

$$
\begin{aligned}
\zeta(t) & =\left(\zeta_{1}(t), \zeta_{2}(t), \cdots, \zeta_{N}(t)\right)^{T} \\
& =\left(\begin{array}{c}
\frac{1}{\sqrt{N}} \mathbf{1}_{N}^{T} \\
S_{k}^{T}
\end{array}\right) C x \\
& =\left(\begin{array}{c}
\frac{1}{\sqrt{N}} \mathbf{1}_{N}^{T} C x \\
S_{k}^{T} C x
\end{array}\right) .
\end{aligned}
$$

Noting that $\mathbf{1}_{N}^{T} C=0$, we have $\zeta_{1}(t)=\frac{1}{\sqrt{N}} \mathbf{1}_{N}^{T} C x=0$. Then

$$
\begin{aligned}
e^{T}\left(\sum_{l=0}^{l_{k}-1} L_{\sigma\left(t_{k}^{l}\right)}\right) e & =\zeta(t)^{T} \operatorname{diag}\left\{\nu_{k}^{1}, \nu_{k}^{2}, \cdots, \nu_{k}^{N}\right\} \zeta(t) \\
& \geq \nu_{k}^{2} \zeta(t)^{T} \zeta(t) \\
& \geq 0
\end{aligned}
$$


From (25) and (28), we have

$$
\lim _{t \rightarrow \infty} \zeta^{T}(t) \zeta(t)=0
$$

This implies

$$
\lim _{t \rightarrow \infty}\left\|e_{i}(t)\right\|=0, i=1,2, \cdots, N .
$$

Therefore, $\lim _{t \rightarrow \infty}\left|x_{i}(t)-x_{j}(t)\right|=0, i, j=1,2, \cdots, N, i \neq j$.

Now, we prove that

$$
\lim _{t \rightarrow \infty}\|\bar{\Theta}(t)\|=0
$$

for any initial condition $\bar{\Theta}(0)$, i.e., for $\forall \epsilon>0$, there exists $t_{\epsilon}>0$ such that $\|\bar{\Theta}(t)\|<\epsilon, \forall t>t_{\epsilon}$.

To prove (31), we first show that: For $\forall \epsilon>0$ and $\hat{T}>0$, there exists $t>\hat{T}$ such that

$$
\left\|\bar{\vartheta}_{i}(t)\right\|<\epsilon, \quad i=1,2, \cdots, N,
$$

for $\forall x(0)$ and $\bar{\Theta}(0)$.

We prove this by contradiction that, for $\forall \epsilon>0$ and some $i \in\{1,2, \cdots, N\}$, a time $t_{\epsilon, 1}$ such that

$$
\left\|\bar{\vartheta}_{i}(t)\right\| \geq \epsilon, \quad \forall t \geq t_{\epsilon, 1}
$$

does not exist.

Consider the time interval $\left[t_{k}, t_{k+1}\right)=\left[t_{k}, t_{k}+T_{k}\right)$ with $T_{0} \leq T_{k} \leq T$ and construct the following function

$$
\Psi(\bar{\Theta}(t), t)=\frac{1}{2}\left[\bar{\Theta}^{T}\left(t+T_{k}\right) \bar{\Theta}\left(t+T_{k}\right)-\bar{\Theta}^{T}(t) \bar{\Theta}(t)\right] .
$$

From the initial condition $x\left(t_{\epsilon}\right)$ and $\bar{\Theta}\left(t_{\epsilon}\right)$, according to (19), (29), and $\lim _{t \rightarrow \infty} V(t)=V(\infty)$, then $\lim _{t \rightarrow \infty} \bar{\Theta}^{T}(t) \bar{\Theta}(t)$ exists and $\lim _{t \rightarrow \infty} \Psi(\bar{\Theta}(t), t)=$ 0 due to $(34)$. Therefore, $\Psi(\bar{\Theta}(t), t)$ is bounded.

Calculating the time derivative of the function $\Psi(\bar{\Theta}(t), t)$ defined in $(34)$ 
at the time instant $t_{k}$, we have

$$
\begin{aligned}
\dot{\Psi}( & \left.\bar{\Theta}\left(t_{k}\right), t_{k}\right) \\
= & \int_{t_{k}}^{t_{k}+T_{k}} \frac{d}{d \tau}\left[\bar{\Theta}^{T}(\tau) \dot{\bar{\Theta}}(\tau)\right] d \tau \\
= & c_{1} \sum_{l=0}^{l_{k}-1} \int_{t_{k}^{l}}^{t_{k}^{l+1}} \frac{d}{d \tau}\left(\bar{\Theta}^{T}(\tau) \Phi(x, \tau) L_{p} e(\tau)\right) d \tau \\
= & \sum_{l=0}^{l_{k}-1} \int_{t_{k}^{l}}^{t_{k}^{l+1}} c_{1}^{2} e^{T}(\tau) L_{p} \Phi^{T}(x, \tau) \Phi(x, \tau) L_{p} \\
& \left.+c_{1} \bar{\Theta}^{T}(\tau) \dot{\Phi}(x, \tau) L_{p}-c c_{1} \bar{\Theta}^{T}(\tau) \Phi(x, \tau) L_{p}^{2}\right\} e(\tau) d \tau \\
& -\sum_{l=0}^{l_{k}-1} \int_{t_{k}^{l}}^{t^{l+1}} c_{1} \bar{\Theta}^{T}(\tau) \Phi(x, \tau) L_{p} \Phi^{T}(x, \tau) \bar{\Theta}(\tau) d \tau \\
\triangleq & I_{1}-I_{2} .
\end{aligned}
$$

Due to the boundedness of $e(t), \bar{\Theta}(t), \Phi(x, t)$, and $\dot{\Phi}(x, t)$, there exists a constant number $M>0$ such that

$$
I_{1} \leq M \sum_{l=0}^{l_{k}-1} \int_{t_{k}^{l}}^{t_{k}^{l+1}}\|e(\tau)\| d \tau .
$$

From Assumption $1, l_{k}, k=1,2, \ldots$, are finite and $\lim _{t \rightarrow \infty}\|e(t)\|=0$, we have

$$
I_{1} \leq \frac{1}{2} c_{1} \alpha \delta_{\min } \epsilon^{2}, \quad \forall t_{k} \geq t_{\epsilon, 2}
$$

Let $U_{p}=\left[\frac{1}{\sqrt{N}} \mathbf{1}_{N}, F_{p}\right] \in \mathcal{R}^{N \times N}$ be an orthogonal matrix, and $F_{p} \in$ $\mathcal{R}^{N \times(N-1)}$ such that

$$
U_{p}^{T} L_{p} U_{p}=\operatorname{diag}\left\{\lambda_{p}^{1}, \lambda_{p}^{2}, \cdots, \lambda_{p}^{N}\right\},
$$

where $\lambda_{p}^{1}=0,0 \leq \lambda_{p}^{2} \leq \cdots \leq \lambda_{p}^{N}$, are the $N$ eigenvalues of $L_{p}$, due to the symmetry of $L_{p}$.

From (38)and $U_{P} U_{P}^{T}=\frac{1}{N} 1_{N} 1_{N}^{T}+F_{p} F_{p}^{T}=I_{N}$, we have $F_{p} F_{p}^{T}=I_{N}-$ $\frac{1}{N} 1_{N} 1_{N}^{T}=C$. Then

$$
\begin{aligned}
L_{p} & =U_{p}\left(\begin{array}{llll}
0 & & & \\
& \lambda_{p}^{2} & & \\
& & \ddots & \\
& & \lambda_{p}^{N}
\end{array}\right) U_{p}^{T} \\
& \geq \delta_{\min } F_{p} F_{p}^{T} \\
& =\delta_{\min } C .
\end{aligned}
$$


Therefore

$$
I_{2} \geq c_{1} \delta_{\min } \sum_{l=0}^{l_{k}-1} \int_{t_{k}^{l}}^{t_{k}^{l+1}} \bar{\Theta}^{T}(\tau) \Phi(x, \tau) C \Phi^{T}(x, \tau) \bar{\Theta}(\tau) d \tau .
$$

By the contradiction, we assume that there exists a time $t_{\epsilon, 1}$ such that (33) is satisfied. From the PE condition (17), we have

$$
I_{2} \geq c_{1} \alpha \delta_{\min } \epsilon^{2}, \quad \forall t_{k}^{l} \geq t_{\epsilon, 1} .
$$

From (35),(37) and (41), we obtain

$$
\dot{\Psi}\left(\bar{\Theta}\left(t_{k}\right), t_{k}\right) \leq-\frac{1}{2} c_{1} \alpha \delta_{\min } \epsilon^{2}<0, \quad \forall t_{k} \geq t_{\epsilon, 3}
$$

with $t_{\epsilon, 3}=\max \left\{t_{\epsilon, 1}, t_{\epsilon, 2}\right\}$, which contradicts the boundedness of $\Psi(\bar{\Theta}(t), t)$. So, (32) holds.

Due to $\lim _{t \rightarrow \infty}\|e(t)\|=0$, we have

$$
\|e(t)\| \leq \sqrt{\frac{1}{2 c_{1} \delta_{\max }}} \epsilon, \quad \forall t \geq t_{\epsilon, 4}
$$

holds for $\forall \epsilon>0$ and some time instant $t_{\epsilon, 4}$.

From (32),

$$
\left\|\bar{\Theta}\left(t_{\epsilon}\right)\right\| \leq \frac{1}{\sqrt{2}} \epsilon
$$

holds for some time instant $t_{\epsilon}>t_{\epsilon, 4}$.

From the initial condition $e\left(t_{\epsilon}\right)$ and $\bar{\Theta}\left(t_{\epsilon}\right)$, according to (19), (43) and (44), we have

$$
\|\bar{\Theta}(t)\| \leq \sqrt{c_{1} \delta_{\max }\left\|e\left(t_{\epsilon}\right)\right\|^{2}+\left\|\bar{\Theta}\left(t_{\epsilon}\right)\right\|^{2}} \leq \epsilon, \quad \forall t \geq t_{\epsilon} .
$$

Thus, (31) holds.

From (30) and (31), it follows that the equilibrium $\operatorname{col}(e(t), \bar{\Theta}(t))=0$ is attractive. Sine (30) and (31) hold uniformly respect to the initial time instant, it follows that $\operatorname{col}(e(t), \bar{\Theta}(t))=0$ is globally uniformly asymptotically stable. Therefore $\lim _{t \rightarrow \infty}\left|x_{i}(t)-x_{j}(t)\right|=0$, for $\forall x_{i}(0) \in \mathcal{R}, i=1,2, \cdots, N$ and $\lim _{t \rightarrow \infty}\left\|\hat{\vartheta}_{i}-\vartheta_{i}\right\|=0$, for $\forall \hat{\vartheta}_{i}(0) \in \mathcal{R}^{m}, i=1,2, \cdots, N$. This completes the proof of the theorem. 
Remark 5. From the proof, it is obvious that all of the variables of the system are bounded. The main result of the paper shows that under the proposed controller (7) and the adaptive law (8), leaderless consensus can be reached, and in the meanwhile, parameter convergence is also ensured. The distribution of the consensus controller (7) and the adaptive law (8) is very important in algorithm design of multi-agent systems. However, the adaptive control algorithm proposed in [19], which considered the leader-following consensus problem, is not purely distributed.

\section{Simulations}

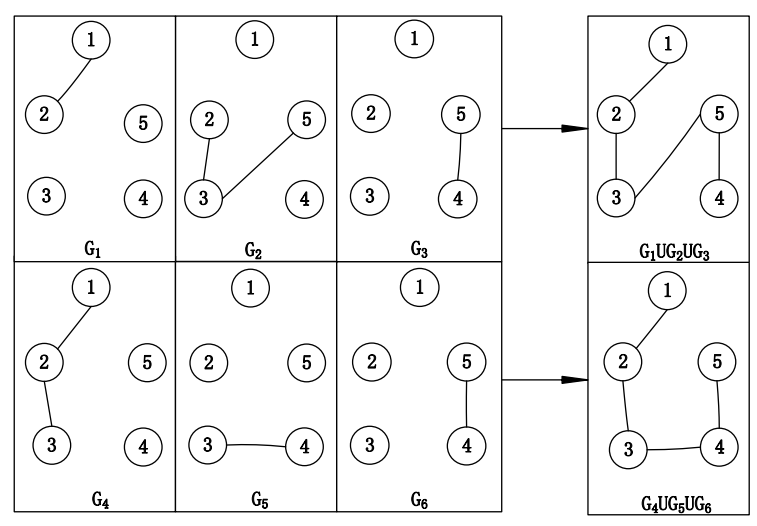

Figure 1: Jointly connected graphs

In this section, an example consisting of five agents is given to validate the theoretical results proposed in this paper. The unknown nonlinear functions are assumed to be parameterized as

$$
f_{i}\left(x_{i}, t\right)=\left[x_{i}(t) \cos (t), x_{i}(t) \sin (t)\right] \vartheta_{i}, \quad i=1,2, \cdots, 5 .
$$

We select $\vartheta_{i}=[-10,10]^{T}, i=1,2, \cdots, 5$, which are assumed to be unknown in our algorithm.

Supposing that all possible switching graphs are $\left\{G_{1}, G_{2}, G_{3}, G_{4}, G_{5}, G_{6}\right\}$ shown in Figure 1. The switching graphs are assumed to switch one time per three time steps according to $G_{1} \rightarrow G_{2} \rightarrow G_{3} \rightarrow G_{4} \rightarrow G_{5} \rightarrow G_{6} \rightarrow G_{1} \cdots$. However, the union of $G_{1} \cup G_{2} \cup G_{3}$ (in 9 time steps) and $G_{4} \cup G_{5} \cup G_{6}$ (in 9 time steps) are jointly connected through some time intervals. Setting the initial states $\operatorname{col}(e(0), \bar{\Theta}(0))=(-1.6,2.3,2.9,0.5,-4.1,4.7,-2.9,0.1,-4.0,3.0,-3.5$, 


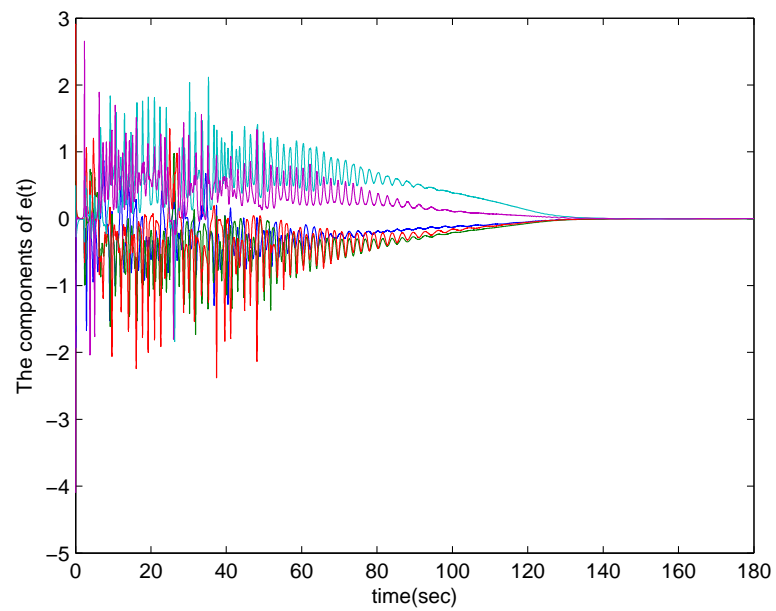

Figure 2: Consensus errors converging to zero under (7) and (8).

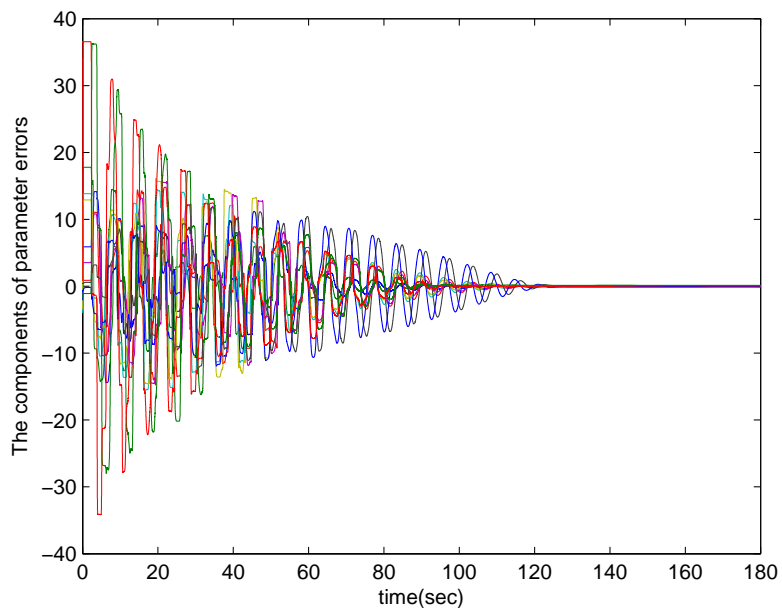

Figure 3: Parameter errors converging to zero under (7) and (8).

$0.4,-2.1,-1.8,0.8)^{T}, c=0.8$ and $c_{1}=100$, the adaptive control algorithms (7) and (8) are simulated in 180 (sec). Figure 2 shows that the consensus errors converge to zero. Figure 3 shows that the parameter errors converge to zero. Figure 4 shows that the components of the estimates of parameters converge to the true parameters -10 and 10 resectively. Figure 5 shows the curves of the control input. The above simulation results validate our 


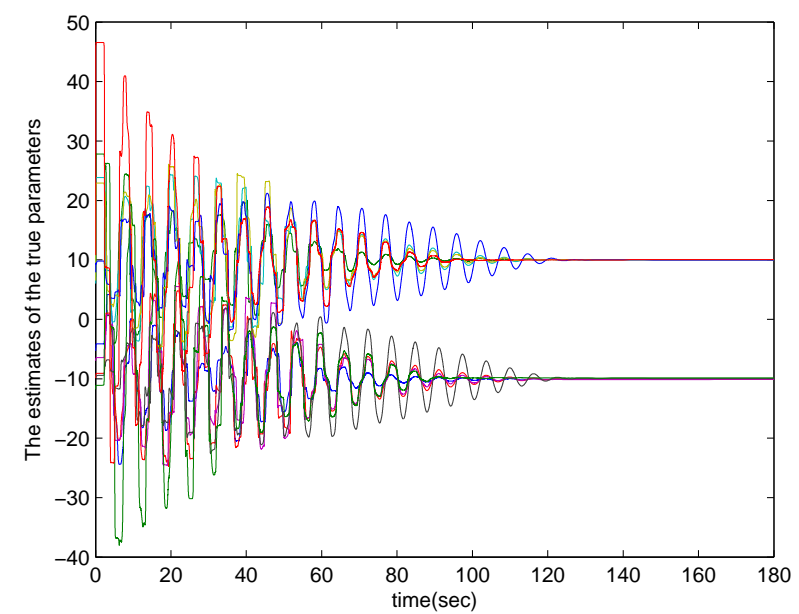

Figure 4: The estimates of the true parameter under (7) and (8).

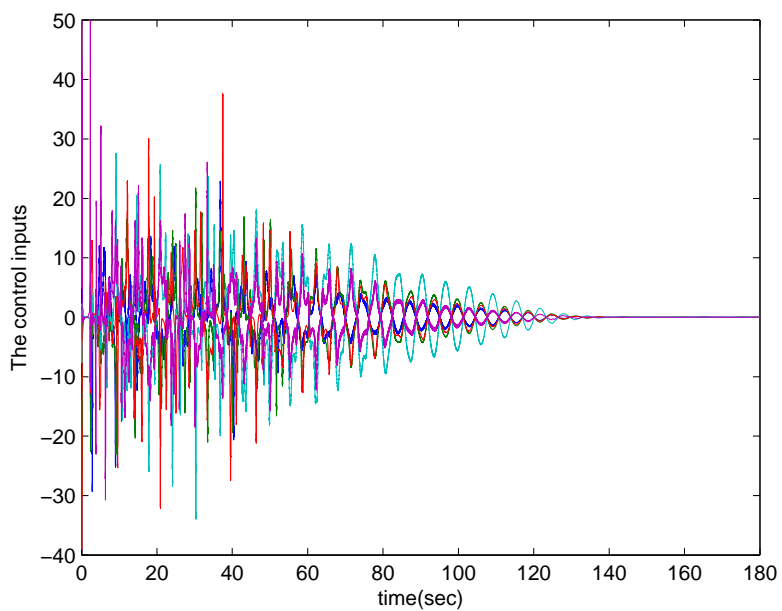

Figure 5: The control input.

theoretical results.

Remark 6. The adaptive algorithms mentioned above [13-22] are mainly divided into two categories. One is using the parameter adaptive method [1320], another is by tuning the coupling strength or network weights [21, 22] adaptively. In this paper, a leaderless consensus algorithm is presented using parameter adaptive method. However, the works [13-20] are all focus on 
leader-following problem.

\section{Conclusions}

In this paper, the leaderless consensus problem of multi-agent systems with uncertainty has been investigated. Leaderless consensus with parameter convergence is ensured by using adaptive design method. We use algebraic graph theory to model the interconnection relations between agents, and the stability analysis has been conducted using the Barbalat's lemma and Lyapunov techniques. For jointly connected graphs, a common matrix $C$ is obtained to derive the PE condition. The joint connectedness of the switching graphs property ensures global uniform asymptotical consensus achieving and the PE condition plays a key role the in the analysis of the global unifor$\mathrm{m}$ asymptotical parameter convergence. The simulation example illustrates the validation of our algorithm. In the future works, we will focus on the following aspects: 1)considering the case of multi-agent systems with directed topologies; 2) searching the sufficient conditions guaranteeing the parameter convergence without the PE condition; 3) searching the relationship between the control performance and parameters associated with jointly connected networks.

\section{Acknowledgements}

This work is supported in part by National Natural Science Foundation (NNSF) of China (61273183, 61374028 and 61304162).

\section{References}

[1] P. Lin, Y. Jia, L. Li, Distributed robust h consensus control in directed networks of agents with time-delay, Systems \& Control Letters 57 (2008) 643-653.

[2] R. Olfati-Saber, R. M. Murray, Consensus problems in networks of agents with switching topology and time-delays, IEEE Transactions on Automatic Control 49 (2004) 1520-1533.

[3] H. Li, C. Ming, S. Shen, W. K. Wong, Event-triggered control for multi-agent systems with randomly occurring nonlinear dynamics and time-varying delay, Journal of the Franklin Institute 351 (2014) 25822599 . 
[4] L. Wang, F. Xiao, Finite-time consensus problems for networks of dynamic agents, IEEE Transactions on Automatic Control 55 (2010) 950955.

[5] P. A. Bliman, Ferrari-Trecate, Giancarlo, Average consensus problems in networks of agents with delayed communications, Automatica 44 (2008) 1985-1995.

[6] Y. G. Sun, L. Wang, G. Xie, Average consensus in networks of dynamic agents with switching topologies and multiple time-varying delays, Systems \& Control Letters 57 (2008) 175-183.

[7] Y. Hong, L. Gao, D. Cheng, J. Hu, Lyapunov-based approach to multiagent systems with switching jointly connected interconnection, IEEE Transactions on Automatic Control 52 (2007) 943-948.

[8] J. Yu, L. Wang, Group consensus in multi-agent systems with switching topologies and communication delays, Systems \& Control Letters 59 (2010) 340-348.

[9] X. Mu, Z. Yang, K. Liu, J. Mu, Containment control of general multiagent systems with directed random switching topology, Journal of the Franklin Institute 352 (2015) 4067-4080.

[10] H. Li, H. Su, Second-order consensus in multi-agent systems with directed topologies and communication constraints, Neurocomputing 173 (2016) 942-952.

[11] X. Mu, X. Xiao, K. Liu, J. Zhang, Leader-following consensus of multiagent systems with jointly connected topology using distributed adaptive protocols, Journal of the Franklin Institute 351 (2014) 5399-5410.

[12] W. Xu, J. Cao, W. Yu, J. Lu, Leader-following consensus of non-linear multi-agent systems with jointly connected topology, IET Control Theory and Applications 8 (2014) 432C-440.

[13] H. Bai, M. Arcak, J. T. Wen, Adaptive design for reference velocity recovery in motion coordination, Systems \& Control Letters 57 (2008) 602-610. 
[14] H. Bai, M. Arcak, J. T. Wen, Adaptive motion coordination: Using relative velocity feedback to track a reference velocity, Automatica 45 (2009) 1020-1025.

[15] Z. G. Hou, L. Cheng, M. Tan, Decentralized robust adaptive control for the multiagent system contensus problem using neural networks, IEEE Transactions on Systems, Man, and Cybernetics 39 (2009) 636-647.

[16] H. Yu, Y. Shen, X. Xia, Adaptive finite-time consensus in multi-agent networks, Systems \& Control Letters 62 (2013) 880-889.

[17] A. Das, F. L. Lewis, Distributied adaptive control for synchronization of unknown nolinear networked systems, Automatica 46 (2010) 2014-2021.

[18] Y. Li, S. Tong, T. Li, Adaptive fuzzy output feedback dynamic surface control of interconnected nonlinear pure-feedback systems, IEEE Transactions on Cybernetics 45 (2015) 138-149.

[19] H. Yu, X. Xia, Adaptive consensus of multi-agents in networks with jointly connected topologies, Automatica 48 (2012) 1783-1790.

[20] W. Chen, C. Wen, S. Hua, C. Sun, Distributied cooperative adaptive identification and control for a group of continuous-time systems with a cooperative pe condition via consensus, IEEE Transactions on Automatic Control 59 (2014) 91-106.

[21] W. Yu, G. Chen, J. Lü, On pinning synchronization of complex dynamical networks, Automatica 45 (2009) 429-435.

[22] H. Li, H. Su, Distributed consensus of multi-agent systems with nonlinear dynamics via adaptive intermittent control, Journal of the Franklin Institute 352 (2015) 4546-4564.

[23] C. D. Godsil, G. Royle, Algebraic Graph Theory, Springer, New York, 2001.

[24] S. Sastry, M. Bodson, Adaptive Control: Stability, Convergence, and Robustness, Prentice-Hall, New Jersey, 1989.

[25] R. Marino, P. Tomei, Nonlinear Control Design - Geometric, Adaptive and Robust, Prentice Hall Europe, 1995. 
[26] H. Yu, J. Sun, Decentralized adaptive consensus in multi-agent networks with jointly connected topologies, Proceedings of 33rd Chinese Control Conference July (2014) 28-32.

[27] K. S. Narendra, A. M. Annaswamy, Stable Adaptive Systems, Englewood Cliffs, NJ: Prentice-Hall, New Jersey, 1989. 